\title{
The Myth of Not Disclosing the Diagnosis of Cancer: Does it Really Protect Elderly Patients from Depression?
}

\author{
Kamile Silay ${ }^{1 *}$, Sema Akinci ${ }^{2}$, Arife Ulas ${ }^{3}$, Yavuz Selim Silay ${ }^{4}$, Muhammed Bulent \\ Akinci $^{5}$, Esin Ozturk ${ }^{6}$, Merve Canbaz ${ }^{6}$, Imdat Dilek ${ }^{7}$, Bulent Yalcin ${ }^{5}$
}

\begin{abstract}
Background: The disclosure of a diagnosis of cancer is complex, particularly in older patients. The aim of this study was to investigate the association between age and not knowing the diagnosis, and its impact on mood. Materials and Methods: The study included 70 patients with various types of solid and hematologic cancer in early stages, which were followed up in an outpatient oncology/hematology clinic in Turkey between January, 2014 and June, 2014. Initially the caregivers of patients were asked whether the patients knew their diagnosis or not. A questionnaire for the Geriatric Depression Scale was then administered to the patients. Patient age, gender, marital status and education level were noted and analyzed with respect to knowing the diagnosis and depression. Results: Of the 70 patients, $40 \%$ of them were female. The mean age was $68.2 \pm 8.9$. The rate of the patients who does not know their diagnosis was $37.1 \%(n=26)$. The overall depression rate with GDS was found $37.1 \%(n=26)$ among the participants. There was no association with knowing the diagnosis $(p=0.208)$ although the association between not knowing the diagnosis and age was significant $(p=0.01)$. Conclusions: In this study we revealed no association between not knowing the diagnosis and depression in elderly patients. Contrary to what some has thought, the patient is not protected from psychological distress by not being informed about the diagnosis. We believe this study and similar ones will help to discuss and further explore patient autonomy, the principle of respect to self-determination and end of life issues in different cultures.
\end{abstract}

Keywords: Elderly - cancer - depression - informing diagnosis - caregiver

Asian Pac J Cancer Prev, 16 (2), 837-840

\section{Introduction}

Cancer is increasing rapidly in the elderly population. Approximately 50 percent of cancers and 70 percent of deaths due to cancer occur in those $\geq 65$ years old, and this is expected to increase (Yancik et al., 2004; Llichtman et al., 2006). Cancer is not only a terminal disease but also a major health problem that leads to mental, emotional and behavioral reactions.

The disclosure of a diagnosis of cancer is complex, particularly in older patients. While the paternalistic approach was popular two decades ago, a non-paternalistic approach is now acceptable. But still in certain cultures, some stigma diseases such as cancer, dementia is not always disclosed to the patient. The belief that "cancer is a terminal disease" is still popular in the society in spite of rapid development and achievements in oncology (Girgis et al., 1998). The disclosure of a terminal disease diagnosis is named "bad news" in the literature (Girgis et al., 1998; Ptacek et al., 1996; Ptacek et al., 2001). This stage is quite difficult not only for a patient but also for other family members and the doctor who is taking care of the patient (Grassi et al., 2000).

One of the study in the literature demonstrated that the preferences of older patients regarding the disclosure of a cancer diagnosis are similar to those of younger patients, as reported by other studies (Parker et al., 2001; Hagerty et al., 2004; Cavanna et al., 2007; Cavanna et al., 2009). However, frequently the physician and the family decide how much information should be given to older patients and traditionally caregivers are less likely to disclose a cancer diagnosis to old patients than to adult or young patients. It must be emphasized that a large proportion of older patients with cancer were not told their diagnosis or not given adequate information about their disease (Kawakami et al., 2001; Cavanna et al., 2009).

There are several reasons behind this "not sharing practice". This is done fundamentally to protect the patient from the distress of diagnosis since depression is common among cancer patients and survivors (Manceton 
Kamile Silay et al

et al., 2012; Zainal et al., 2013). Also the thought of; 'lack of patient's ability to understand the information' and 'family's wish that the patient not be informed of the truth' were among the other major reasons for non-disclosure. As the society structure changes, interesting trends in these practices may be captured. Therefore, to understand in a society of emerging and developing nation, we have designed this cross sectional study. We aim to investigate the association between age and not knowing the diagnosis and its impact on mood of elderly cancer patients.

\section{Materials and Methods}

\section{Subjects}

The study included 70 patients with various types of solid and hematologic cancer in early stages, which were followed up in outpatient oncology/hematology clinic between January, 2014 and June, 2014. The inclusion criteria were age older than 65 who has good support system and Eastern Cooperative Oncology Group (ECOG) performance status is equal to or less than 2 . The exclusion criteria were poor performance status and history of psychiatric disease or depression.

\section{Measurements}

Demographic data; age, sex, educational level, marital status, support systems were recorded. A survey administered to the primary caregiver of the patient. They were asked whether the cancer diagnosis was disclosed the patient or not. If not the reason of not disclosing the diagnosis was questioned.

\section{Depression status}

Geriatric Depression Scale was administered to all patients. The Geriatric Depression Scale (GDS), first created in 1982 (Yesavage, et al., 1982) has been tested and used extensively with the older population. A Short Form GDS consisting of 15 questions was developed in 1986. Questions from the Long Form GDS which had the highest correlation with depressive symptoms in validation studies were selected for the short version. This instrument has been studied in multiple settings (Montorio et al., 1996; Rinaldi et al., 2003). Scores of 0-4 are considered normal, depending on age, education, and complaints; 5-8 indicate mild depression; 9-11 indicate moderate depression; and 12-15 indicate severe depression.

\section{Data analysis}

Patients were divided according to their age in three groups to those between 55 to 65,65 to 75 and older than 75 years old. Patients who completed elementary school accepted as educated. The cancer patients who had GDA scores equal to 5 or more were classified as the possibly depressed group, and those who had GDA scores less than 5 were classified as the non-depressed group.

Then the association between age, marital status, education level and knowing the diagnosis and depression were analyzed with Pearson's chi square test. The association between the variables were considered significant when $p<0.05$. For the statistics of the study SPSS.20 software was used.
Table 1. The Analysis of 'Not Knowing the Diagnosis'

\begin{tabular}{|c|c|c|c|c|}
\hline & & $\mathrm{N}(\%)$ Do & ot know diagr & \\
\hline & & & $\mathrm{N}(\%)$ & $\mathrm{p}$ value \\
\hline Age & $55-65$ & $24(34.3 \%)$ & $2(8.3 \%)$ & 0.01 \\
\hline & $65-75$ & $31(44.3 \%)$ & $14(45.2 \%)$ & \\
\hline & $>75$ & $15(21.4 \%)$ & $10(66.7 \%)$ & \\
\hline Education & Illiteracy & $31(44.3 \%)$ & $16(51.6 \%)$ & 0.045 \\
\hline Gender & Female & $28(40.0 \%)$ & $13(46.4 \%)$ & 0.215 \\
\hline & Male & $42(60.0 \%)$ & $13(31 \%)$ & \\
\hline Depression & & $26(37.1 \%)$ & $7(26.9 \%)$ & 0.208 \\
\hline
\end{tabular}

\section{Results}

There were 70 patients, $40 \%$ of them were female. The mean age of patients was $68.2 \pm 8.9$. Patients distribution according to age was $55-65=34.3 \%(n=24), 65-75=44.3 \%$ $(n=31), 75=21.4 \%(n=15)$. Thirty one patients were uneducated $(44.3 \%)$; thirty nine patients were educated $(55.7 \%)$. Most patients were married $81.4 \%(n=57)$..

The rate of the patients who does not know their diagnosis was $37.1 \%(n=26)$. The depression rate with GDS was found $37.1 \%(n=26)$ among the participants.

The association between age and not knowing the diagnosis is found statistically significant. The rate of not knowing diagnosis in age groups 55-65, 65-75 and $>75$ were $8.3 \%, 45.2 \%, 66.7 \%$ respectively $(\mathrm{p}=0.01)$. The association between not knowing the diagnosis and depression was not found statistically significant $(\mathrm{p}=0.208)$. But the association between not knowing the diagnosis and education was statistically significant $(p=0.045)$. (Table 1). The education level was found lower in female patients $(\mathrm{p}=0.008)$. No association was found between age and depression $(\mathrm{p}=0.422)$.

\section{Discussion}

This is an important study providing insight into beliefs and preferences of the caregivers and evaluating the mood of elderly patients about diagnosis of a serious disease. In this study we revealed that as the patient ages, the diagnosis of cancer is concealed from the patient. However, no association was found between 'not knowing the diagnosis' and 'depression' in elderly patient contrary to general belief.

The disclosure of a diagnosis of cancer is a complex issue, particularly in older patients since cancer is usually considered synonymous with death, pain and suffering (Powe et al., 2003; Tavoli et al., 2007). A large proportion of older patients with cancer were not told their diagnosis or not given adequate information about their disease (Kawakami et al., 2001; Cavanna et al., 2009). Patients and families interpret the diagnosis of cancer as equivalent to death and therefore caregivers may prefer not to disclose the diagnosis (Montazeri et al., 2002; Vahdaninia et al., 2003; Tavoli et al., 2007). The rate of patients who does not know their diagnosis was found $37.1 \%$ in our study which is similar to literature. The age and knowing diagnosis was found inversely related. The caregivers of patients who preferred not to disclose the diagnosis to their patients were questioned about the reason of their decision. Majority of the caregivers (76\%) indicated that, they want 
to protect their patient from the stress and anxiety of the cancer diagnosis. Caregivers believe the word of "cancer "may cause pyschological distress such as anxiety and depression in the patient and affect negatively their quality of life. The rest of the caregivers believe that, the patient would not understand the information and treatment plan.

One of the studies in the literature including gastrointestinal cancer patients showed lower levels of anxiety and depression in patients who did not know their cancer diagnosis (Tavoli et al., 2007). Although the incidence and severity of psychological distress are reported to be less than that observed in younger patients, approximately one-third of older patients with cancer experience psychological distress. This most typically takes the form of depression. The prevalence of clinically significant depression is estimated to be 3 to 25 percent in the older population with cancer (Kua et al., 2005; Vinokur et al., 2007).

The depression rate was found $37,1 \%$ in this study. However, we did not show any association between knowing diagnosis and depression in older cancer patients. Depression is not a normal consequence of aging. The rates of depression for community-dwelling older adults range from 2 percent to about 10 percent for patients with minor depression (Beekman et al., 1999). Rates of depression are higher for older adults with comorbid medical illness and in general medical settings. Depressed mood may be the first symptom of some medical conditions affecting the elderly including stroke, diabetes, cancer, hypothyroidism, and coronary disease (Polsky et al., 2005). Our findings suggest that depression is common among elderly patients and but this might be due to other factors such as recent onset of physical illness, severity of physical illness, functional disability, poorly treated pain and multiple illnesses rather than knowing the diagnosis.

Knowing the diagnosis and take part in the management plan is an essential right of a patient. Evidence suggests that sensible disclosure of diagnosis and prognosis is important and satisfaction with information-giving is associated with a better quality of life (Annunziata et al., 1998; Tavoli et al., 2007). The principle of respect to self-determination means that an individual has a right and an ability to make decisions according to his/her belief, values and life plan (Aydın et al., 2003). Therefore, physicians have a legal and ethical responsibility to provide adequate information to the patient for processing the information and make appropriate decisions (Berg et al., 2001). In this way patients have the right to choose how to manage their disease and plan their last days of their life as they wish.

Another important finding revealed in our study is the association between illiteracy and not knowing the diagnosis. Female patients found less educated than males. The less educated people may not be query about the diagnosis or/and caregivers may think 'lack of patient's ability to understand the information'. Further prospective, larger studies are needed to evaluate this issue.

In conclusion, as the population continues to age the diagnosis of cancer is gradually increasing. Elderly patient and cancer combination will be part of our life on a daily basis, therefore the right diagnosis and treatment as well as accurately informing and engaging the patient and caregiver is critical. In this study we revealed that as the patient ages the diagnosis of catastrophic nature such as cancer is concealed from the patient.

However, no association was found between not knowing the diagnosis and depression in elderly patient. Contrary to what some has thought, the patient is not protected from psychological distress by not informing about the diagnosis. Actually with this practice one might say that, basic right of patients such as self- determination, making decisions according to his/her belief, values and life plan is ignored with presumably good intentions.

We believe this study and similar ones will help to discuss and further explore the patient autonomy, the principle of respect to self-determination and end of life issues in different cultures.

\section{References}

Annunziata MA, Foladore S, Magri MD, et al (1998). Does the information level of cancer patients correlate with quality of life? A prospective study. Tumori, 84, 619-23.

Aydin E (2003). The principle of respect for autonomy of the patient in medical ethics. Erciyes Med J, 25, 92-7.

Beekman AT, Copeland JR, Prince MJ (1999). Review of community prevalence of depression in later life. $\mathrm{Br} J$ Psychiatry, 174, 307.

Berg JW, Appelbaum PS, Lidz CW, et al (2001). Informed consent: legal theory and clinical practice, $2^{\text {nd }}$ edition, Oxford University Press, New York.

Cavanna L, Anselmi E, Lazzaro A, et al (2007). Cancer diagnosis disclosure in a northern Italian hospital. Report on 312 consecutive cancer patients. Tumori, 93, 290-1

Cavanna L, Di Nunzio C, Seghini P, et al (2009). Elderly cancer patients' preferences regarding the disclosure of cancer diagnosis. Experience of a single institution in Italy. Tumori, 95, 63-7.

Girgis A, Sanson-Fisher RW (1998). Breaking bad news. 1: Current best advice for clinicians. Behav Med, 24, 53-9.

Grassi L, Gritti P, Rigatelli M, et al (2000). Psychosocial problems secondary to cancer: an Italian multicentre survey of consultation-liaison psychiatry in oncology. Italian consultation-liaison group. Eur J Cancer, 36, 579-85

Hagerty RG, Butow PN, Ellis PA, et al (2004). Cancer patient preference for communication of prognosis in the metastatic setting. J Clin Oncol, 22, 1721-30.

Kawakami S, Arai G, Ueda K, et al (2001). Physicians' attitudes towards disclosure of cancer diagnosis to elderly patients: a report from Tokyo, Japan. Arch Gerontol Geriatr, 33, 29-36,

Kua J (2005). The prevalence of psychological and psychiatric sequelae of cancer in the elderly-how much do we know? Ann Acad Med Singapore, 34, 250.

Lichtman SM (2006). Therapy insight: Therapeutic challenges in the treatment of elderly cancer patients. Nat Clin Pract Oncol, 3, 86.

Maneeton B, Maneeton N, Mahathep P (2012). Prevalence of depression and its correlations: a cross-sectional study in Thai cancer patients. Asian Pac J Cancer Prev, 13, 2039-43.

Montazeri A, Vahdaninia M, Haji-Mahmoodi M, et al (2002). Cancer patient education in Iran: a descriptive study. Support Care Cancer, 10, 169-73.

Montorio I, Izal M (1996). The geriatric depression scale: a review of its development and utility. Int Psychogeriatr, 8, 103.

Parker PA, Baile WF, de Moor C, et al (2001). Breaking bad 
news about cancer: patients' preferences for communication. J Clin Oncol, 19, 2049-56.

Polsky D, Doshi JA, Marcus S, et al (2005). Long-term risk for depressive symptoms after a medical diagnosis. Arch Intern Med, 165, 1260.

Powe BD, Finne R (2003). Cancer fatalism: the state of the science. Cancer Nurs, 26, 454-65.

Ptacek JT, Eberhardt TL (1996). Breaking bad news. A review of the literature. JAMA, 276, 496-502.

Ptacek JT, Ptacek JJ, Ellison NM (2001). "I"m sorry to tell you ..." physicians" reports of breaking bad news. J Behav Med, 24, 205-17.

Rinaldi P, Mecocci P, Benedetti C, et al (2003). Validation of the five-item geriatric depression scale in elderly subjects in three different settings. J Am Geriatr Soc, 51, 694.

Tavoli A, Mohagheghi MA, Montazeri A, et al (2007). Anxiety and depression in patients with gastrointestinal cancer: does knowledge of cancer diagnosis matter. BMC Gastroenterol, 7, 28.

Yancik R, Ries LA (2004). Cancer in older persons: an international issue in an aging world. Semin Oncol, 31, 128.

Yesavage JA, Brink TL, Rose TL, et al (1982). Development and validation of a geriatric depression screening scale: a preliminary report. J Psychiatr Res, 17, 37-49.

Vahdaninia MS, Montazeri A (2003). Cancer patient education in Iran: attitudes of health professionals. J Iranian Inst Health Sci Res Persian Payesh, 2, 259-65.

Vinokur AD, Threatt BA, Vinokur-Kaplan D, et al (1990). The process of recovery from breast cancer for younger and older patients. Changes during the first year. Cancer, 65, 1242.

Zainal NZ, Nik-Jaafar NR, Baharudin A, Sabki ZA, Ng CG (2013). Prevalence of depression in breast cancer survivors: a systematic review of observational studies. Asian Pac J Cancer Prev, 14, 2649-56. 